\title{
Percutaneous Repair of an Aortic Pseudoaneurysm Status Post-Surgical Repair of Stanford Type A Dissection
}

\author{
Brinder S Kanda, MD, FACC, FSCAI ${ }^{*}$, Nicholas E Hoyt ${ }^{(D)}$, John Haas, MD, Houman Tavaf-Motamen, \\ MD, Ronald Morford, MD, Tina Wesley, BSN, Kenneth Saum, MD and Michael MacCormac, MD
}

Centra Health Heart and Vascular Center, USA

*Corresponding author: Dr. Brinder S Kanda, Centra Health Heart and Vascular Center, 2410 Atherholt Road, Lynchburg, VA 24501, USA, Tel: 434-200-5252, Fax: 434-200-5252

\begin{abstract}
A middle-aged Caucasian woman presented with an ascending aortic pseudoaneurysm (AAP) located at the suture site of her previous surgical dissection repair. The pseudoaneurysm was located along the anterior aspect of the ascending thoracic aorta, above the right coronary cusp. We demonstrate successful percutaneous repair of the pseudoaneurysm using a ventricular septal occluder (VSO) and discuss the significance of this procedure for future cardiovascular care.
\end{abstract}

\section{Keywords}

Aorta, Ascending aortic pseudoaneurysm, Ventricular septal occluder, Left anterior descending artery, Right coronary artery, Computed tomography angiography, Transesophageal echocardiography

\section{Abbreviations}

AAP: Ascending Aortic Pseudoaneurysm; VSO: Ventricular Septal Occluder; LAD: Left Anterior Descending Artery; RCA: Right Coronary Artery; CTA: Computed Tomography Angiography; TEE: Transesophageal Echocardiography

\section{Introduction}

A 56-year-old Caucasian woman with a history of active tobacco abuse, systemic lupus erythematosus, and hypothyroidism underwent emergent surgical repair of a Stanford type A aortic dissection with a 28 $\mathrm{mm}$ Gelweave graft. Her aortic valve was intact and did not require repair. She also underwent empiric aortocoronary bypass with saphenous vein grafting to the left anterior descending artery (LAD). Her re- covery was slow, and her hospital course was prolonged, but she was eventually discharged home. One-month routine follow-up computer topography angiography (CTA) of her aorta demonstrated that a pseudoaneurysm had developed at the suture site along the anterior aspect of the ascending thoracic aorta, above the right coronary cusp (Figure $1 \mathrm{~A}$ and Figure $1 \mathrm{~B}$ ). The patient was referred to the heart clinic for further management.

Differential diagnoses included a true aneurysm, pseudoaneurysm, and mycotic aneurysm. CTA was utilized to distinguish pseudoaneurysm from true aneurysm based on the visualization of disruption of the intimal and medial layers of the ascending aorta. Lack of infectious signs and symptoms, plus negative blood cultures, served to exclude a mycotic aneurysm. At this time, the patient reports nonspecific fatigue and atypical nonexertional chest pain symptoms.

\section{Methods}

The patient was evaluated by our Structural Heart Team, and her computed topography (CT) scan was reviewed at our Multidisciplinary Structural Conference. Her CT demonstrated a pseudoaneurysm located at the proximal suture site, along the anterior aspect of the ascending thoracic aorta, just above the right coronary cusp (Figure $1 \mathrm{~A}$ and Figure 1B). The neck of the pseudoaneurysm was $9.5 \mathrm{~mm}$ (Figure 2A), the depth was $16.5 \mathrm{~mm}$ (Figure 2B), and the side-to-side diameter was $21.2 \mathrm{~mm}$ (Figure 2C). The distance of the right coronary

Citation: Kanda BS, Hoyt NE, Haas J, Tavaf-Motamen H, Morford R, et al. (2020) Percutaneous Repair of an Aortic Pseudoaneurysm Status Post-Surgical Repair of Stanford Type A Dissection. Int J Clin Cardiol 7:166. doi.org/10.23937/2378-2951/1410166

Accepted: February 27, 2020; Published: February 29, 2020

Copyright: (C) 2020 Kanda BS, et al. This is an open-access article distributed under the terms of the Creative Commons Attribution License, which permits unrestricted use, distribution, and reproduction in any medium, provided the original author and source are credited. 
artery (RCA) ostium from the neck of the pseudoaneurysm sac was $7.5 \mathrm{~mm}$.

Our team decided that the patient was not a good candidate for redo-sternotomy due to her significant frailty, and the prior difficult recovery from her initial operation. We proceeded with percutaneous repair, as the anatomical positioning seemed favorable. We elected to use a $12 \mathrm{~mm}$ Amplatzer muscular VSO (Abbott Laboratories), as this provided a safe landing zone above the RCA, based on three-dimensional CT reconstruction.
After a rigorous Structural team review, we had an open discussion with the patient and her family. She understood that this was an off-label procedure and wished to proceed. We accessed the patient's bilateral common femoral arteries using a modified Seldinger technique under fluoroscopic and ultrasound guidance. An 8-French sheath was placed on the right groin, and a 5 -French sheath was placed on the left groin. A 9 -French left femoral venous sheath was also placed using modified Seldinger technique in the anticipation of requiring intracardiac echocardiography. The patient was anticoagulated with systemic heparin. We kept the activated

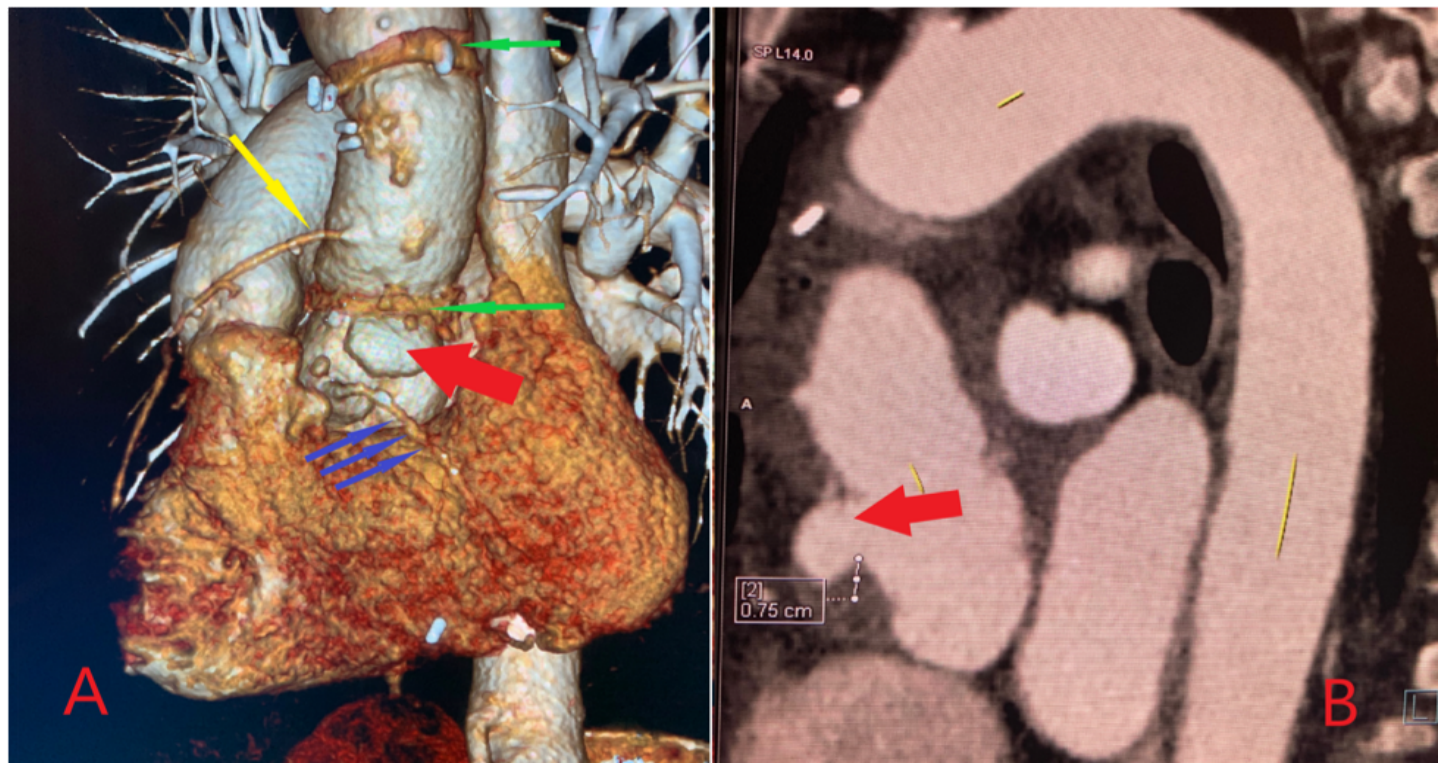

Figure 1: A) Solid green arrows: Proximal and distal suture lines of the Gelweave repair graft; Solid yellow arrow: Saphenous vein graft to the left anterior descending artery; Solid blue arrows: Right coronary artery; Solid red arrow: Location of the ascending aortic pseudoaneurysm at the suture line; B) Solid red arrow: Location of the ascending aortic pseudoaneurysm at the suture line.

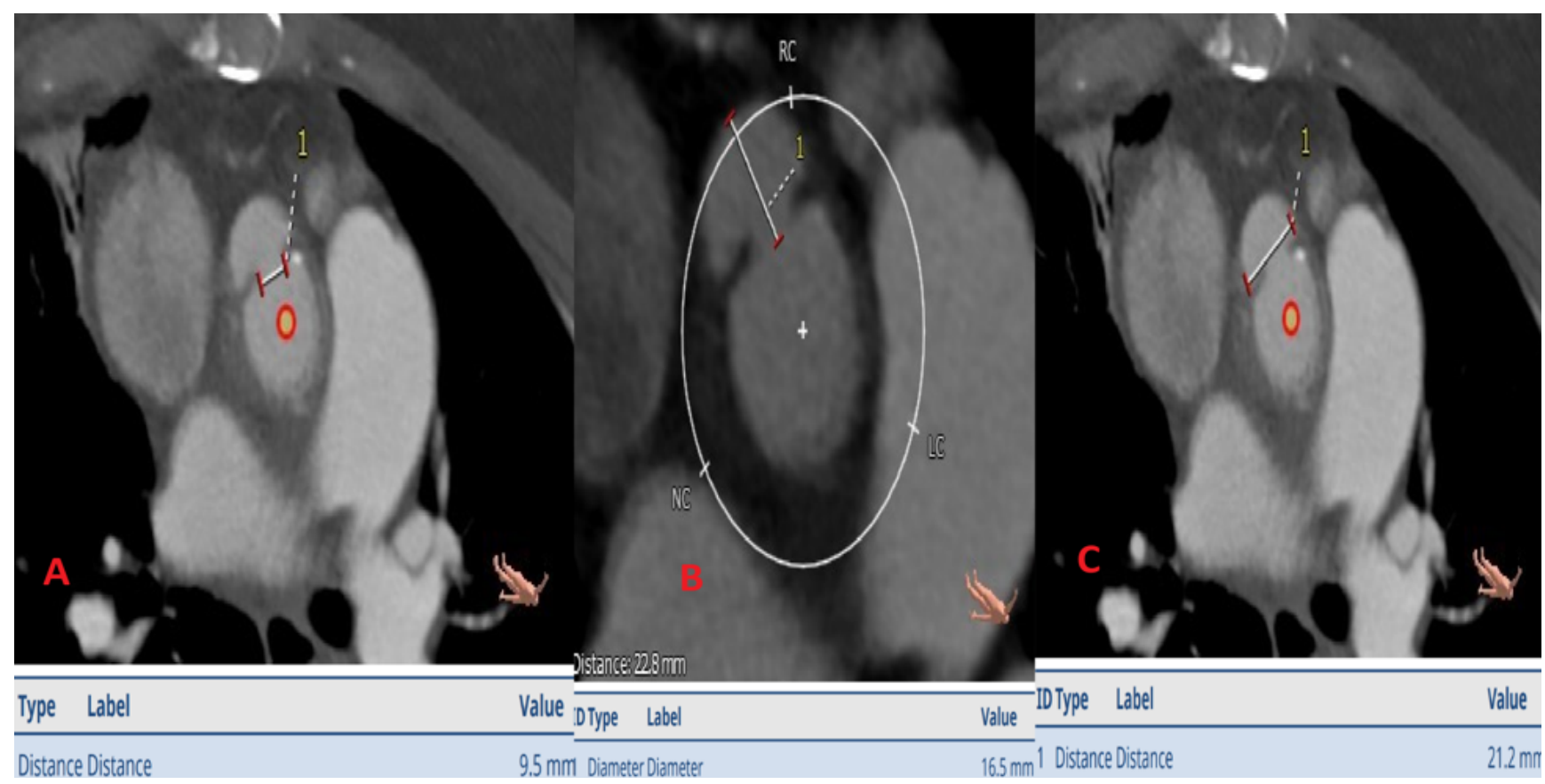

Figure 2: A) Demonstrating the neck measurement of the pseudoaneurysm; B) Demonstrating the depth measurement of the pseudoaneurysm; C) Demonstrating the side-to-side (width) measurement of the of the pseudoaneurysm. 
clotting time between 250 and 300 throughout the entire case. The intracardiac echocardiogram probe was advanced, and baseline images were taken. The images were not optimal, so we decided to switch to transesophageal echocardiogram (TEE) imaging. A Judkins R4 catheter was used to engage the pseudoaneurysm and angiography was performed. Fortunately, the Judkins R4 catheter engaged the pseudoaneurysm well. We used the heat gun to shape the 8-French TorqVue ${ }^{\mathrm{TM}}$ delivery catheter (Abbott Laboratories) to seat well. We choose a $12 \mathrm{~mm}$ Amplatzer ventricular septal defect occluder, as the outer disc diameter was $4 \mathrm{~mm}$ on each side of the occluder waist. This was done to avoid right coronary occlusion. Under transesophageal echocardiography and fluoroscopic guidance, the $12 \mathrm{~mm} \mathrm{Am-}$ platzer muscular ventricular septal defect occluder was deployed in good position. Right coronary angiography was performed to ensure that there was no occlusion. The right coronary was patent and was easily engageable. There was no evidence of compromise of the RCA ostium, which was located in close proximity to the inferior margin of the device. Aortic root angiography was performed with a pigtail catheter that showed appropriate closure of the pseudoaneurysm, and there was no evidence of contrast reflux from the pseudoaneurysm to the aorta (Figure 3A, Figure 3B and Figure $3 \mathrm{C}$ ). The aortic valve was intact without any compromise of leaflet mobility. The following morning, the patient underwent TEE that demonstrated a well seated plug and normal aortic valve functioning.

She had a closely monitored follow-up in our structural heart clinic. Her follow up CT demonstrated a well seated occluder device and successful closure of her AAP (Figure 4 and Figure 5).

\section{Results}

The patient was seen in our office two weeks status post pseudoaneurysm repair. A limited echocardiogram showed the muscular VSD occluder to be in a good position. There was no compromise of the aortic valve or nearby structures. The patient denied any signs of hemolysis. She had no hematuria or difficulty urinating. Her chest pain had resolved. Physical exam findings were normal and showed a well healed midline scar.

The most recent chest CTA, three weeks status post procedure, demonstrated successful repair of the small AAP associated with the original surgery (Figure 5A, Figure 5B and Figure 5C). Persistent mild pericardial effusion was present as well as cardiomegaly.

\section{Discussion}

Aortic pseudoaneurysms occur when the intima and media of a vessel are disrupted. They are enclosed by adventitia and the surrounding structures of the mediastinum [1]. Without intervention, aortic pseudoaneurysms are usually problematic, as they are likely to expand in size, compressing and ultimately degrading surrounding structures while also acting as a source of persistent infection and systemic embolism [2].

Ascending aortic pseudoaneurysms develop from cannulation sites from aortic perfusion catheters, origin of saphenous vein grafts conduits, cardioplegia cannulation sites, and aortotomy sites from aortic valve replacement during pulmonary bypass. Ascending aortic pseudoaneurysms are difficult to treat, and

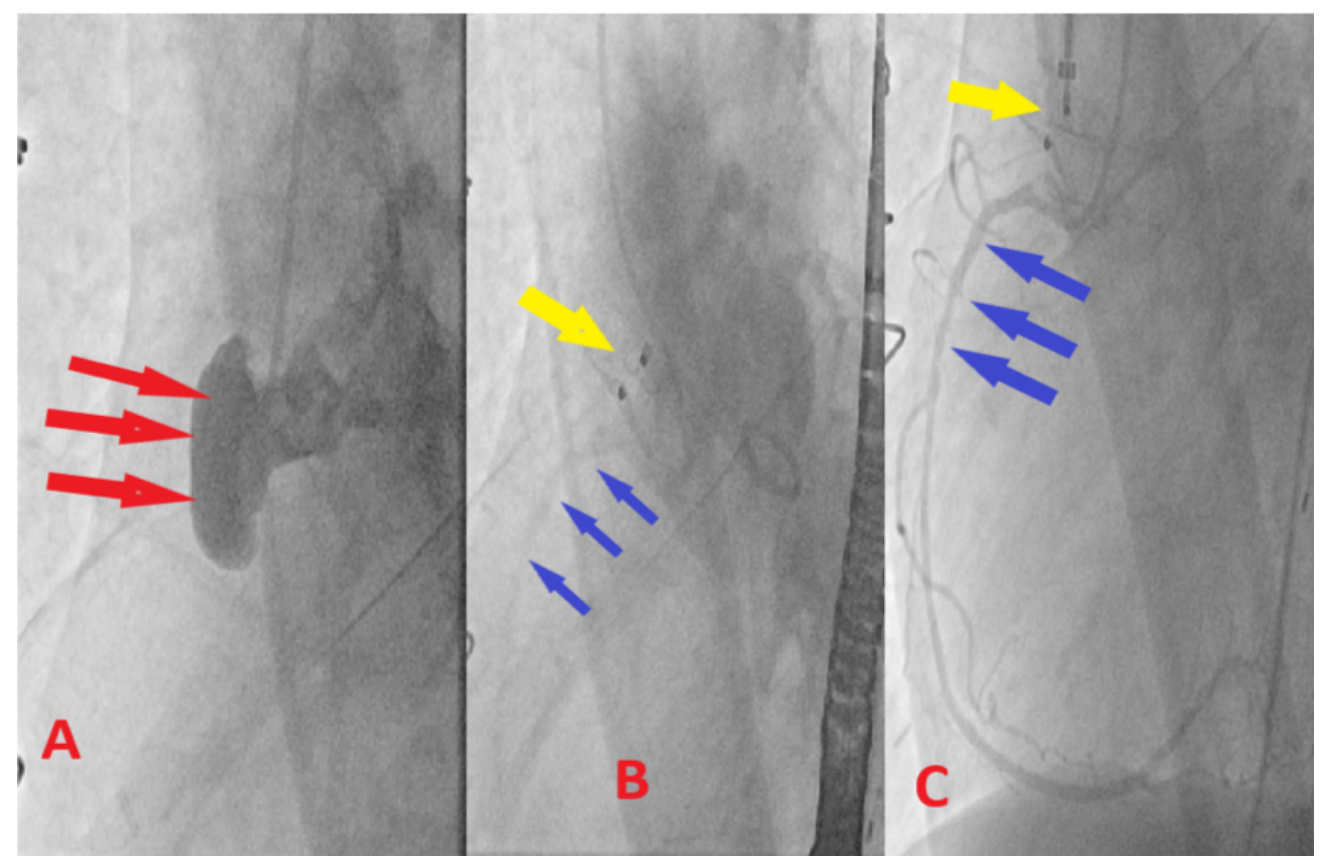

Figure 3: A) Red arrows: Demonstrating intraoperative angiography of the AAP; B) Yellow arrow: Demonstrating well seated occluder device; Blue arrows: Patent RCA; C) Selective engagement of the RCA, while occluder is in place. 


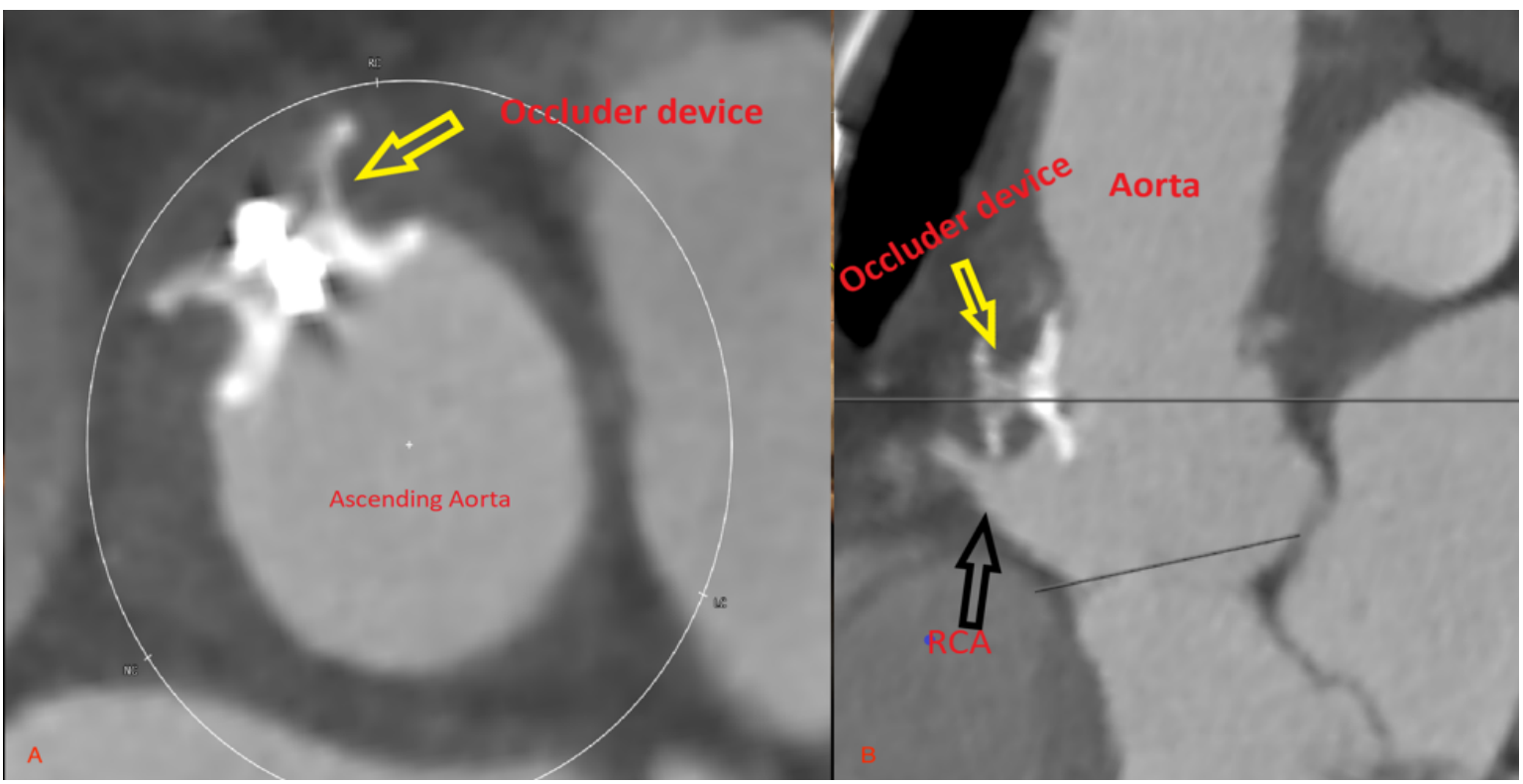

Figure 4: Postoperative 2D CT demonstrating a well seated occluder device and patent RCA. Yellow arrows: Well seated occluder device; Black arrow: Patent right coronary artery.
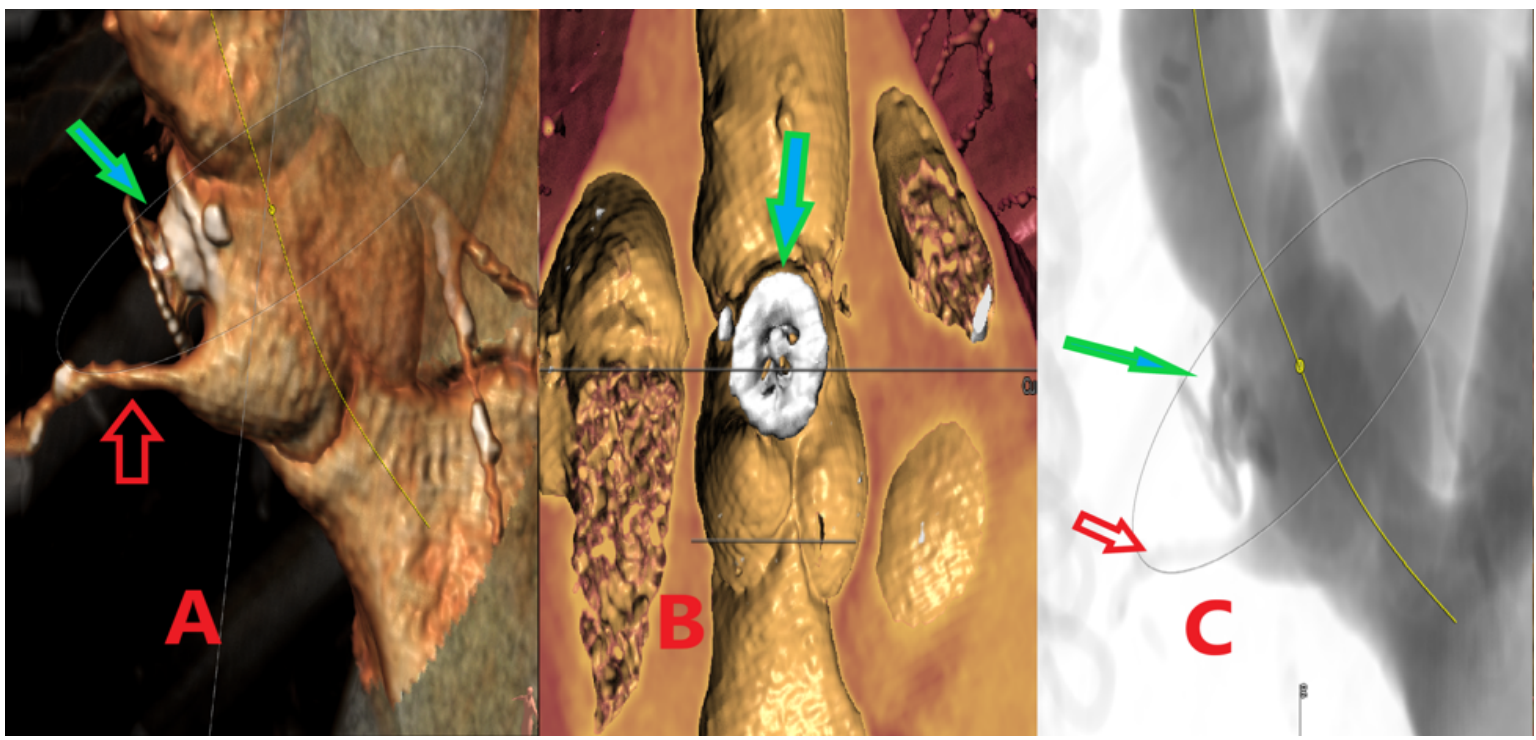

Figure 5: Postoperative 3D computer tomography demonstrating a well seated occluder device and patent right coronary artery.

Blue/green arrows: Well seated occluder device; Red arrows: Patent right coronary artery.

surgical techniques to repair ascending aortic pseudoaneurysms have shown higher rates of morbidity and mortality than an endovascular approach [3].

The incidence rates of ascending aortic pseudoaneurysms have been reported to be as low as $0.5 \%$, but one surveillance imaging series found a higher incidence rate in patients following cardiac or aortic surgery [4]. A study of ascending aortic pseudoaneurysms between 1980 and 2014 discovered that most ascending aortic pseudoaneurysm cases were surgically treated with a Dacron graft, Hemashield graft, pericardial patch, interrupted stitches, or a hybrid procedure in combination with stent grafts. Endovascular approach was less common [5].
Surgical treatment of this condition carries significant morbidity and mortality. Due to variable location and different anatomical challenges, there is no standard percutaneous approach or dedicated device. Depending on the location, sometimes stent grafts can be utilized. Stent grafts are usually designed for the descending, distal arch and abdominal aortic pathology. The course of the ascending aorta and arch, along with the variability of the great vessels and coronary takeoffs, make this anatomy difficult to navigate [6]. Vascular plugs, atrial septal occluders, and ventricular septal occluders can be used, depending on the anatomical requirements.

Our case study demonstrates a percutaneous ap- 
proach for successfully treating the AAP utilizing the VSD occluder device. Due to proximity of RCA, we were careful in our device choice. There is no "one size fits all" approach for treating this rare complication. In certain cases, redo sternotomy may still be required. A careful, comprehensive, and multidisciplinary team approach can benefits patients when planning the correction of an AAP.

\section{Conclusion}

Aortic pseudoaneurysms are a rare complication following aortic surgery. If left untreated, they can expand and compress other structures, rupture, or even become a source of infection. While surgical intervention to repair the AAP is most common, it carries a higher rate of mortality and morbidity than an endovascular approach. The complex anatomy of the ascending aorta and aortic arch make it difficult to apply endovascular grafting techniques, but our case study has demonstrated that it is still possible to utilize an endovascular approach with an Amplatzer muscular VSD occluder device. Correct placement was absolutely necessary as our patient's pseudoaneurysm was located within $1 \mathrm{~mm}$ of the RCA. This location increased the risk of RCA dissection or occlusion. By using an endovascular approach, we reduced the patient's likelihood of morbidity and mortality with a redo sternotomy.
The authors report no financial relationships or conflict of interest regarding the content herein.

All authors agree with the information included in the manuscript and its submission.

\section{References}

1. Dumont E, Carrier M, Cartier R, Pellerin M, Poirier N, et al. (2004) Repair of aortic false aneurysm using deep hypothermia and circulatory arrest. Ann Thorac Surg 78: 117-121.

2. Katsumata T, Moorjani N, Vaccari G, Westaby S (2000) Mediastinal false aneurysm after thoracic aortic surgery. Ann Thorac Surg 70: 547-552.

3. Makaroun MS, Dillavou ED, Kee ST, Sicard G, Chaikof E, et al. (2005) Endovascular treatment of thoracic aortic aneurysms: Results of the phase II multicenter trial of the Gore TAG thoracic endoprosthesis. J Vasc Surg 41: 1-9.

4. Mesana TG, Caus T, Gaubert J, Collart F, Ayari R, et al. (2000) Late complications after prosthetic replacement of the ascending aorta: What did we learn from routine magnetic resonance imaging follow-up? Eur $\mathrm{J}$ Cardiothorac Surg 18: 313-320.

5. Quevedo HC, Santiago-Trinidad R, Castellanos J, Atianzar $\mathrm{K}$, Anwar A, et al. (2014) Systematic review of interventions to repair ascending aortic pseudoaneurysms. Ochsner $\mathrm{J}$ 14: 576-585.

6. Yuri K, Yamaguchi A, Hori D, Nemoto K, Kawaguchi S, et al. (2010) A fenestrated stent graft for endovascular repair of an ascending aortic pseudoaneurysm. Ann Vasc Dis 3: 228-231. 